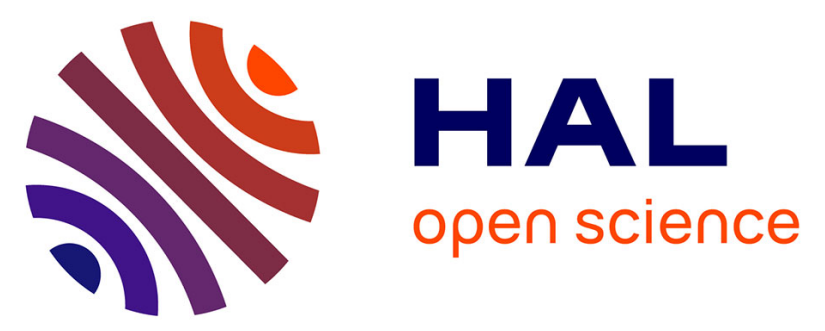

\title{
Sub-microscopic Plasmodium falciparum infections in matched peripheral, placental and umbilical cord blood samples from asymptomatic Congolese women at delivery
}

Yvon Mbouamboua, Koukouikila-Koussounda Félix, Ntoumi Francine, Selorme Adukpo, Michael Kombo, Christevy Vouvoungui, Jacques van Helden, Simon Charles Kobawila

\section{- To cite this version:}

Yvon Mbouamboua, Koukouikila-Koussounda Félix, Ntoumi Francine, Selorme Adukpo, Michael Kombo, et al.. Sub-microscopic Plasmodium falciparum infections in matched peripheral, placental and umbilical cord blood samples from asymptomatic Congolese women at delivery. Acta Tropica, 2019, 193, pp.142-147. 10.1016/j.actatropica.2019.03.001 . hal-02078131

\section{HAL Id: hal-02078131}

https://hal-amu.archives-ouvertes.fr/hal-02078131

Submitted on 13 Dec 2019

HAL is a multi-disciplinary open access archive for the deposit and dissemination of scientific research documents, whether they are published or not. The documents may come from teaching and research institutions in France or abroad, or from public or private research centers.
L'archive ouverte pluridisciplinaire HAL, est destinée au dépôt et à la diffusion de documents scientifiques de niveau recherche, publiés ou non, émanant des établissements d'enseignement et de recherche français ou étrangers, des laboratoires publics ou privés. 
Sub-microscopic Plasmodium falciparum infections in matched peripheral, placental and umbilical cord blood samples from asymptomatic Congolese women at delivery

Yvon Mbouambouaa,b, Félix Koukouikila-Koussoundaa,b, Francine Ntoumia,b,c* , Selorme Adukpo $^{\mathrm{c}, \mathrm{d}}$, Michael Kombo ${ }^{\mathrm{a}}$, Christevy Vouvoungui ${ }^{\mathrm{a}, \mathrm{b}}$, Jacques van Helden ${ }^{\mathrm{e}}$ and Simon Charles Kobawilab

a Fondation Congolaise pour la Recherche Médicale, Brazzaville, Republic of Congo

b Faculty of Sciences and Techniques, University Marien Ngouabi, Brazzaville, Republic of Congo

c Institute for Tropical Medicine, University of Tübingen, Tübingen, Germany

d Immunology Department, Noguchi Memorial Institute for Medical Research, University of Ghana, Legon, Ghana

e Aix-Marseille Univ, INSERM UMR_S 1090, Theory and Approaches of Genome Complexity (TAGC), F-13288 Marseille, France

${ }^{*}$ Corresponding author at Fondation Congolaise pour la Recherche Médcale Villa D6, Campus WHO/AFRO, Djoué, Brazzaville, Rep of Congo

Email address: fntoumi@fcrm-congo.com (F.Ntoumi)

${ }^{1} E$-mail addresses: fridyvon@gmail.com (Y. Mbouamboua), fntoumi@fcrm-congo.com (F. Ntoumi), felixkoukouikila@yahoo.fr (F. Koukouikila-Koussounda), sadukpo812@gmail.com (S. Adukpo), michael.kombo@yahoo.fr (Michael Kombo), vjchristevy@gmail.com (C. Vouvoungui), Jacques.vanHelden@univ-amu.fr (J. Helden), kobawila.simon@gmail.com (S. Kobawila)

Graphical abstract 


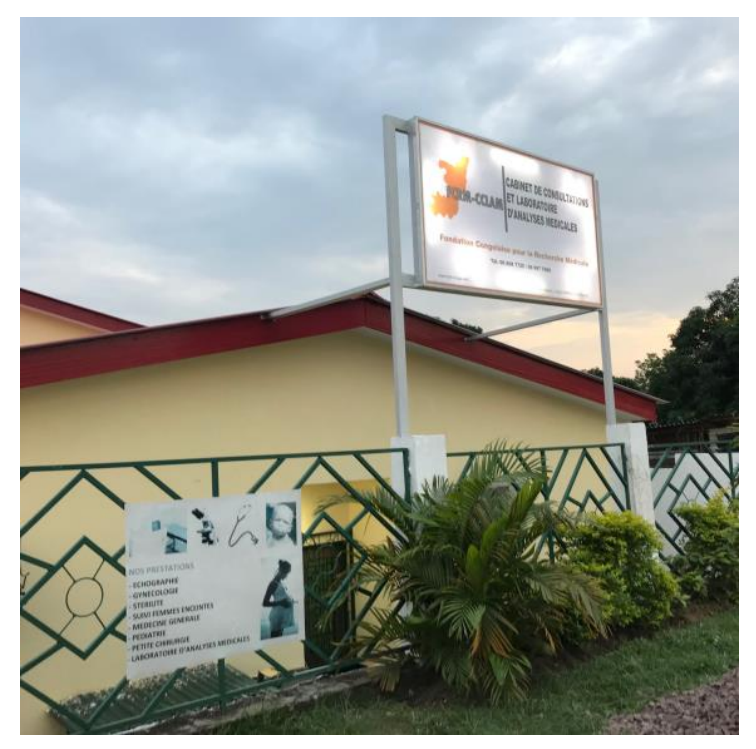

\section{Heading Highlights}

- High prevalence of sub-microscopic infection at delivery.

- High genetic diversity of Plasmodium falciparum strains in Congo that varies according to peripheral, placental and umbilical cord blood.

- Reduction of multiplicity of infections.

\section{Abstract}

In malaria-endemic areas, most pregnant women are susceptible to asymptomatic Plasmodium falciparum infections. We present here the results of a cross-sectional study conducted in Madibou, a southern district of Brazzaville in the Republic of Congo, between March 2014 and April 2015. The main aim was to characterize $P$. falciparum infections. Blood samples corresponding to peripheral, placental and cord from 370 asymptomatic malaria women at delivery were diagnosed for plasmodium infection by thick blood smears (microscopic infection). Sub-microscopic infection was detected by PCR, using the MSP-2 gene as marker. Microscopic infections were detected in peripheral, placental and cord blood samples with a prevalence of respectively $7.3 \%(27 / 370), 2.7 \%(10 / 370)$ and $0 \%$. The negative samples were submitted to sub-microscopic detection, with respective prevalence of $25.4 \%$ (87/343), 16.7\% (60/360) and 9.4\% (35/370) $(\mathrm{P}<0.001)$. We further 
investigated the genetic diversity of the parasite by characterizing MSP2 allelic families 3D7 (24 distinct alleles) and FC27 (20 distinct alleles). The total number of alleles for these two families were 31, 25 and 19 in peripheral, placental and cord samples respectively. The 3D7 MSP-2 was the predominant allelic family. The multiplicity of infections (MOI) in peripheral (mean 1.4 \pm 0.01 ; range 1-4), placental (mean 1.2 \pm 0.01 ; range 1-3) and cord samples $(1.4 \pm 0.01$; range $1-3)$ were similar $(P=0.9)$ and are unaffected by age, gravidity or sulfadoxine-pyrimethamine. These results shown a high prevalence of sub-microscopic infection and a high genetic diversity of Plasmodium falciparum strains in Congo. Age, gravidity and doses of preventive treatment based on sulfadoxine-pyrimethamine do not interfere with the multiplicity of infections.

Keywords: asymptomatic pregnant women, Plasmodium falciparum, genetic diversity, multiplicity of infections, Congo.

\section{Introduction}

Malaria infection during pregnancy is a serious public health issue in sub-Saharan Africa with approximately 30 million pregnant women being at risk of pregnancy-associatedmalaria (Dellicour et al., 2010). The symptoms and complications of malaria infection during pregnancy may vary according to the level of malaria transmission in different geographical areas and the level of acquired immunity (Okell et al., 2009). In hightransmission areas, most infections during pregnancy are asymptomatic but still result in poor pregnancy outcome (Steketee et al., 1996; Mockenhaupt et al., 2000; Nosten et al., 2004). A high proportion of individuals living in malaria-endemic areas harbour $P$. falciparum parasites undetectable by microscopy - therefore called sub-microscopic infection - which can nevertheless be identified using molecular tools like PCR (Snounou et al., 1993). Sub-microscopic infection is common during pregnancy and at delivery (Cottrell et al., 2015). To reduce the burden of malaria infections in pregnant women, the World Health Organization (WHO) has recommended a package of interventions, including the use of long-lasting insecticidal nets (LLINs) and administration of sulfadoxine- 
pyrimethamine (SP) as intermittent preventive treatment of malaria during pregnancy (IPTp) (WHO, 2005). In the Republic of Congo, a malaria-endemic country in Central Africa with perennial transmission (Trape and Zoulani, 1987), the administration of IPTpSP ( 2 or 3 doses) during pregnancy has been in place since 2006. The study conducted in asymptomatic pregnant women in Brazzaville revealed a reduction of the prevalence of $P$. falciparum infection (Ntoumi et al., 2016). However, high resistance to SP has been found in parasites collected from Congolese pregnant women (Koukouikila-Koussounda et al., 2015). The development of an effective vaccine is the one of the major challenges of the fight against malaria. However, this approach is limited by the polymorphic nature of Plasmodium (Mayengue et al., 2004; Francine et al., 2016; Guitard et al., 2010; Cohee et al., 2016). The susceptibility of pregnant women to malaria is explained by the presence of parasite strains that specifically bind to the chondroitin sulphate A (CSA) present in the placental tissue (Fried and Duffy, 1996; Duffy and Fried, 1999). Investigating of genotype and the circulating parasites in pregnant women is interesting for the malaria vaccine (Genton et al., 2002; Takala et al., 2007; Takala and Plowe, 2009). Several previous studies on the antigenic diversity of $P$. falciparum matched in peripheral, placental and umbilical cord blood have shown varied results in different country. Some studies have shown a parasitic overlap found for each of these compartments while others have revealed a subpopulation of peripheral parasites sequestered in the placenta (Kassberger et al., 2002; Mayengue et al., 2004; Fadlelseed et al., 2017; Guitard et al., 2010; Cohee et al., 2016). Recent published work conducted in the same area reported 29 MSP-2 alleles in isolates from peripheral blood of pregnant women with asymptomatic $P$. falciparum infection at antenatal visits, suggesting a wide diversity of the $P$. falciparum population (Francine et al., 2016). Therefore, the present cross-sectional study was conducted to characterize $P$. falciparum infections matched in peripheral, placental and cord blood among Congolese women at delivery receiving 1, 2 or more doses of sulfadoxinepyrimethamine. 


\section{Materials and methods}

\subsection{Study site}

The study was carried out at Madibou Integrated Health Center, located in the semi-urban area of Makélékélé district, Southern part of Brazzaville, the capital of the Republic of Congo. This area with about 6.000 inhabitants is situated along the Congo River, where malaria transmission is intense and continuous throughout the year. The entomological inoculation rate is approximately 23 infective bites/person/year (Trape and Zoulani, 1987). P. falciparum is the predominant malaria species and Anopheles gambiae s.s the main mosquito vector.

\subsection{Study population}

Between March 2014 and April 2015, a total of 370 pregnant women were recruited at the maternity ward. The inclusion criteria were as follows: signed informed consent obtained from the participant at admission before delivery, no episodes of clinical malaria during the last two weeks, no notion of fever at least 48 hours before enrolment and an axillary temperature $\leq 37.5^{\circ} \mathrm{C}$ during the examination. Socio-demographic data were recorded using a structural questionnaire and from medical records.

\subsection{Ethics}

The study was approved by the Institutional Ethics Committee of the Fondation Congolaise pour la Recherche Médicale (ethical clearance No 001/CEl/FCRM/2012). Written informed consent was obtained from all pregnant women before enrolment.

\subsection{Blood collection and treatment of samples}

A total volume of $5 \mathrm{ml}$ of peripheral blood was collected before delivery, $5 \mathrm{ml}$ placental intervillous and umbilical cord blood were collected within 30 min after delivery into EDTA tubes for the detection of and quantification of malaria parasites. Thick blood smears were 
prepared for each blood compartment, stained with $10 \%$ Giemsa solution for $15 \mathrm{~min}$, and examined by an experienced microscopist. Trophozoites were counted concomitantly with 200 leukocytes, and expressed in number of asexual parasites per $\mu \mathrm{L}$ of blood, assuming the leukocyte count of $8.000 / \mu \mathrm{L}$ of blood. The rest of the blood was spun to separate cells from plasma and stored separately at $-20^{\circ} \mathrm{C}$ for future use.

\subsection{DNA extraction and characterization of $P$. falciparum isolates}

Genomic DNA was extracted separately from peripheral, placental and cord blood using the QIAmp DNA Blood Mini kit (QIAGEN GmbH, Hilden, Germany) according to manufacturer instructions. Parasite isolates were genotyped for $P$. falciparum using the nested PCR technique, targeting the highly polymorphic locus MSP-2 central region as previously described (Francine et al., 2016). The initial amplifications were followed by individual nested PCR reactions, using specific primers for FC27 and 3D7 allelic families of the MSP-2 gene. Allele-specific positive controls (3D7 and Dd2) and DNA-free water as a negative control were included in each set of reactions. Each PCR product was stained with Sybr Green, loaded on a $2 \%$ agarose gel, and visualized under ultraviolet transillumination. Various alleles were determined by their amplicon sizes and the corresponding allele-specific primers used. The sizes of PCR product were estimated using a 100 bp DNA ladder marker (Invitrogen, Karlsruhe, Germany). It was assumed that one band represented one amplified PCR fragment derived from a single copy of $P$. falciparum MSP-2 gene. Alleles in each family were considered as one if fragment sizes were within a $20 \mathrm{bp}$ interval. The number of different MSP-2 fragments identified per isolate was considered as the minimum number of parasite genotypes present in this isolate. The amplified PCR products from the different blood compartments for the one woman were run in the same gel to ensure a better comparison.

\subsection{Case definitions}

Asymptomatic $P$. falciparum infection was defined as the presence of malaria parasites in blood in the absence of any clinical symptom. Peripheral, placental and cord blood isolates were grouped into a) $P$. falciparum microscopy positive infection; b) $P$. falciparum infection 
that were negative by microscopy but detected by PCR (sub-microscopic infection); c) negative individuals who were negative by both microscopy and PCR. The genetic diversity of MSP-2 alleles was determined as the total number of different MSP-2 fragments amplified. The multiplicity of infection (MOI) was defined as the mean number of $P$. falciparum genotypes per infected woman. Gravidity was grouped as paucigravidae (First and second full-term pregnancies), and multigravidae (3rd full term pregnancies).

\subsection{Statistical analysis}

All statistical analyses were performed using $R$ (version 3.5.1) under the RStudio environment (1.1.456). To evaluate the impact of different factors (age, gravidity, SP), we applied non-parametric tests, since the multiplicity of infections takes discrete values, and does thus not comply with the normality assumption required for parametric tests. For twogroup comparisons (age, gravidity) we applied Wilcoxon test. For multi-group comparisons (number of SP doses) we ran a Kruskal-Wallis test. For multi-group comparisons between compartments (prevalence of infection; allelic diversity), we applied the Kruskal-Wallis test. The statistical significance threshold was set to $P<0.05$.

\section{Results}

\subsection{Characteristics of the study population}

From March 2014 to April 2015, a total of 370 women were enrolled in the study. The characteristics of the women are shown in Table 1. 
Table 1. Characteristics of the Congolese women at delivery. The age and gestational age are given as mean plus or minus standard deviation. $\mathrm{N}=$ number of women in each group.

\begin{tabular}{ll}
\hline Parameters & Values $(N=370)$ \\
\hline Mean age \pm SD (years) & $25.9 \pm 6.45$. \\
Mean gestational age \pm SD (weeks) & $38.1 \pm 1.9$ \\
Gravidity, $n(\%)$ & \\
$\quad$ Paucigravidae & $152(41 \%)$ \\
$\quad$ Multigravidae & $218(59 \%)$ \\
Number of IPTp-SP doses & \\
No & $60(16.2 \%)$ \\
1 dose & $106(28.6)$ \\
2 doses & $95(25.7 \%)$ \\
3 doses & $112(30.3 \%)$ \\
\hline
\end{tabular}

3.2. Prevalence of $P$. falciparum asymptomatic infection

Overall, matched peripheral, placental and cord blood samples from all study participants were analysed. The prevalence of $P$. falciparum microscopic infection in peripheral blood was $7.3 \%$ and that of placental blood was $2.7 \%$ (Table 2). Not a single sample was found microscopic positive in cord blood (Table 2). The prevalence of $P$. falciparum submicroscopic infections was $25.4 \%$ in peripheral blood, $16.7 \%$ in placental blood and $9.4 \%$ in cord blood (Table 2). All the microscopy-positive peripheral and placental blood samples were positive by PCR. The overall (microscopy-positive and sub-microscopic infections put together) prevalence of $P$. falciparum sub-microscopic infection was $30.8 \%$ in peripheral blood and $19.4 \%$ in placental blood (Table 2).

Table 2. Prevalence of MSP-2 P. falciparum infections in Congolese women at delivery. $\mathrm{N}$ : number of women recruited in this study. For each woman, we collected three blood samples corresponding for peripheral, placenta and cord. 


\begin{tabular}{lllll}
\hline $\mathrm{N}=370$ & Peripheral, $\mathrm{n}(\%)$ & Placenta, $\mathrm{n}(\%)$ & Cord, $\mathrm{n}(\%)$ & $P$ \\
\hline Infections & & & & \\
Microscopy & $27 / 370(7.9)$ & $10 / 370(2.7)$ & $0 / 370(0.0)$ & 0.005 \\
Sub-microscopy & $87 / 343(25.4)$ & $60 / 360(16.7)$ & $35 / 370(9.5)$ & $<0.001$ \\
Total PCR & $114 / 370(30.8)$ & $70 / 370(19.4)$ & $35 / 370(9.5)$ & $<0.001$ \\
\hline
\end{tabular}

Out of $87(25.4 \%)$ of women with peripheral sub-microscopic infections, $9(4.6 \%)$ had placental infections and 11 (12.6\%) had infections in cord. Out of $60(16.7 \%)$ of women with placental sub-microscopic infections, $4(6.7 \%)$ samples were matched in cord. Furthermore, 62 (71.3\%), $42(70 \%)$ and 15 (42.8\%) positive samples were detected exclusively in peripheral, placental and cord respectively. Overall, 5 cases of $P$. falciparum sub-microscopic infections matched in peripheral, placental and cord samples (Fig. 1).

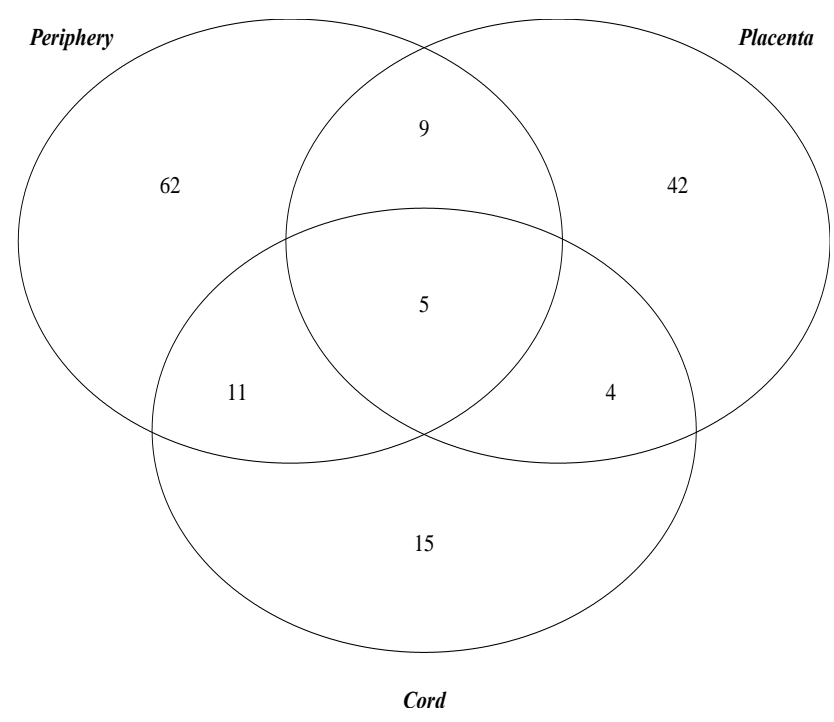

Fig. 1: Sub-microscopic P. falciparum infections in Congolese women at delivery matched in peripheral, placental and cord blood.

\subsection{Distribution of P. falciparum MSP-2 alleles}

With regard to the genetic diversity, the distribution of different MSP-2 alleles is shown in Table 3. A total, 30,25 and $19(P=0.29)$ alleles were detected respectively in the peripheral, placental and cord samples. The 3D7 family was predominant whatever sample 
types. The mixed 3D7 and FC27 alleles were more prevalent in peripheral samples (26.3\%) (Table 3).

Table 3. Genetic diversity of $\boldsymbol{P}$. Falciparum MSP-2 gene. Total Nb. of alleles: total number of 3D7 and FC27 alleles of each compartment.

\begin{tabular}{|c|c|c|c|c|c|c|c|c|}
\hline \multirow[b]{2}{*}{ Alleles } & \multicolumn{4}{|c|}{$\mathrm{Nb}$. of samples (\%) } & \multirow{2}{*}{$\begin{array}{l}\text { Fragment size range (bp) } \\
\text { Peripher Placenta Cord } \\
\text { y }\end{array}$} & \multicolumn{3}{|c|}{ Total Nb. of alleles (\%) } \\
\hline & Periphery & Placenta & Cord & $P$ & & Periphery & Placenta & Cord \\
\hline $3 \mathrm{D7}$ & $62(54.4)$ & $42(60)$ & $16(45.7)$ & $<0.001$ & $180-680 \quad 140-600 \quad 120-480$ & $16(51.6)$ & $14(56)$ & $12(63.2)$ \\
\hline FC27 & $22(19.3)$ & $20(28.6)$ & $11(31.4)$ & 0.14 & $260-620 \quad 260-540 \quad 240-520$ & $14(48.4)$ & $11(44)$ & $7(36.8)$ \\
\hline $3 \mathrm{D} 7+\mathrm{FC} 27$ & $30(26.3)$ & $8(11.4)$ & $8(22.9)$ & $<0.001$ & & & & \\
\hline Total & $114(100)$ & $70(100)$ & $35(100)$ & $<0.001$ & & $30(100)$ & $25(100)$ & $19(100)$ \\
\hline
\end{tabular}

The 3D7 and FC27 alleles identified in the paired peripheral / placental, peripheral / cord and placental / cord blood samples were compared. The proportion of samples matched with identical and partially concordant alleles is shown in Figure 2, which summarizes the number of alleles found in peripheral, placental and cord blood. Some samples revealed the same alleles in the three compartments. In other cases, one or both samples had alleles not detected in the other ones. 
A

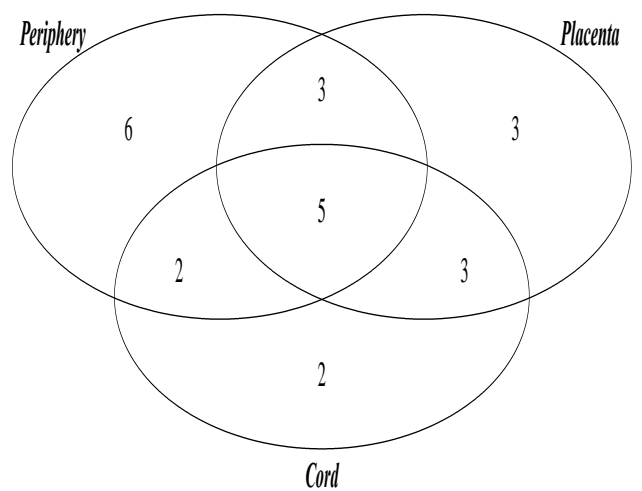

$B$

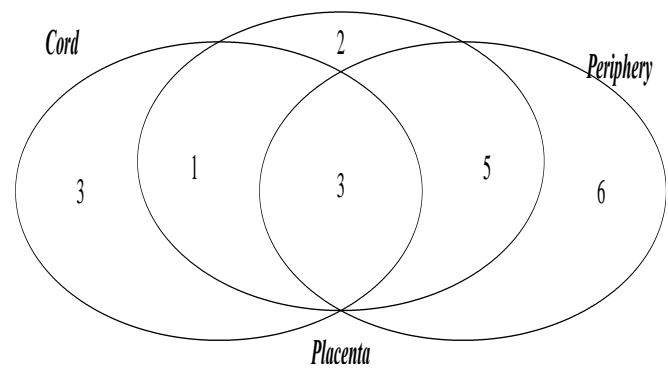

Fig. 2: comparison of $P$. falciparum MSP-2 alleles detected in paired peripheral, placental and cord blood from Congolese women at delivery. (A) 3D7 family. (B) FC27 family.

\subsection{Multiplicity of $P$. falciparum infections}

The $\mathrm{MOI}$ was $1.4 \pm 0.01$ (ranged 1-4 clones) in peripheral, $1.2 \pm 0.01$ (range 1-3 clones) in placental and 1.4 \pm 0.01 (ranged 1-3 clones) in cord samples. Of 114 infected samples from peripheral blood, 110 (96.5\%) contained single and 40 (35.1\%) multiples infections. Of the 70 infected samples from placental blood, 46 (65.7\%) harboured single and 24 $(34.3 \%)$ multiples infections. In cord, $32(91.4 \%)$ of 35 infected samples contained multiples infections and $3(8.6 \%)$ samples have single infection. The MOI was neither affected by maternal age, nor gravidity or SP doses administered (Table 4). 
Table 4: Multiplicity of $\boldsymbol{P}$. falciparum infections according to age of mother, gravidity and SP doses administered. Prevalence of infection determined by PCR, including positive peripheral, placental and/or cord samples. Multiplicity of infections $=$ mean number of $P$. falciparum genotypes per infected woman. The columns labelled $P$ indicate the $P$-value of a mean comparison test, based on either the Wilcoxon test or Kruskal-Wallis test depending on the number of groups. The null hypotheses are explicated in the column header.

Multiplicity of P. falciparum infections (range) in the different compartments

\begin{tabular}{lllllll}
\hline & Periphery & $P$ & Placenta & $P$ & Cord & $P$ \\
\hline Overall & $1.4(1-4)$ & & $1.2(1-3)$ & & $1.4(1-3)$ &
\end{tabular}

\begin{tabular}{|c|c|c|c|c|c|c|}
\hline \multicolumn{7}{|c|}{ Age (year). Wilcoxon test with $H_{0}: m(<18)=m(\geq 18)$. } \\
\hline$<18$ & $1.4(1-2)$ & \multirow{2}{*}{0.79} & $1.3(1-2)$ & \multirow{2}{*}{0.78} & $2.0(1-2)$ & \multirow{2}{*}{0.88} \\
\hline$\geq 18$ & $1.4(1-4)$ & & $1.2(1-3)$ & & $1.4(1-3)$ & \\
\hline \multicolumn{7}{|c|}{ Gravidity. Wilcoxon test with $H_{0}: m($ Paucigravidae $)=m($ Multigravidae $)$. } \\
\hline Paucigravidae & $1.4(1-3)$ & \multirow{2}{*}{0.26} & $1.1(1-3)$ & \multirow{2}{*}{0.94} & $1.3(1-3)$ & \multirow{2}{*}{0.26} \\
\hline Multigravidae & $1.3(1-4)$ & & $1.3(1-3)$ & & $1.4(1-3)$ & \\
\hline \multicolumn{7}{|c|}{ IPTp-SP. Kruskal-Wallis test with $H_{0}: m($ no dose $)=m(1$ dose $)=m(2$ doses $)=m(3$ doses $)$} \\
\hline No & $1.2(1-2)$ & \multirow{4}{*}{0.39} & $1.1(1-2)$ & \multirow{4}{*}{0.39} & $1.7(1-3)$ & \multirow{4}{*}{0.39} \\
\hline 1 dose & $1.4(1-3)$ & & $1.2(1-3)$ & & $1.7(1-3)$ & \\
\hline 2 doses & $1.6(1-4)$ & & $1.1(1-2)$ & & $1.6(1-3)$ & \\
\hline 3 doses & $1.1(1-3)$ & & $1.3(1-3)$ & & $1.1(1-3)$ & \\
\hline
\end{tabular}

\section{Discussion}

The current study is the first of its kind to evaluate the frequency of sub-microscopic $P$. falciparum infection among malaria asymptomatic Congolese women at delivery. The main findings of this study are the high prevalence of sub-microscopic infection at delivery, high diversity of parasites circulating in the population, and differential patterns of MSP-2 allelic 
families in matched peripheral, placental and umbilical cord parasite isolates from even the same individual.

The prevalence of sub-microscopic $P$. falciparum infection in the peripheral and placental blood was higher than microscopic infection in all compartments. In the cord compartment, the $P$. falciparum infection was found only by PCR. This indicates that the threshold of detection of $P$. falciparum by microscopy in pregnant women strongly under-estimates the actual infection rates (Francine et al., 2016; Mockenhaupt et al., 2000). Thus, reliance on microscopy may lead to miss diagnosis and failure to avert adverse effect of malaria during pregnancy (Mockenhaupt et al., 2000; Ntoumi et al., 2013; Ntoumi et al., 2016).

The prevalence of sub-microscopic infection was generally high at delivery. This suggests that pregnant women remained at increased risk of new infections during the late period of their pregnancies after receiving IPTp-SP, which is mostly administered during the second trimester. This increased danger may explain why despite the risk of infection being significantly reduced among delivered women who received 2 or 3 doses of IPTp-SP, there were still about $20 \%$ who harboured sub-microscopic infection. The proportion of submicroscopic infection in our study is quite small compared to those reported from Gabon (Tshibola et al., 2014). This difference might be due to the transmission intensity of the parasite at the different study sites and possibly differential host factors such as a degree of immunity that might restrict parasites growth to microscopically undetectable levels. Alternatively, these low-density infections may represent recently acquired infections, which could reach detectable levels by microscopy if infections were not treated.

All microscopic placental blood infections were microscopically detectable in the peripheral blood. However, 42 women with sub-microscopic infection of the placental blood showed no evidence of peripheral blood infection. During pregnancy, parasites expressing a ligand for chondroitin sulphate $A$ tend to adhere to placenta and sequester in this unique compartment (Fried and Duffy, 1996) and are rarely detected in the peripheral blood. This might have accounted for the observation made in these 42 participants. The prevalence of placental $P$. falciparum infection was lower than peripheral, which is consistent with some of the previous reports (Omalu et al., 2012; Hviid et al., 2011) but not with others 
(Mayengue et al., 2004; Ouédraogo et al., 2012). The high prevalence of infection in peripheral than placenta blood may explain the new $P$. falciparum infections in third trimester of pregnancy.

Asymptomatic $P$. falciparum infection is highly prevalent in malaria endemic regions, which raises a serious concern about the efforts at eliminating the parasite (Riley et al., 2001). Particularly, sub-patent malaria is still transmissible and will complicate the elimination of malaria in high-transmission regions (Sturrock et al., 2013). Most studies suggested that exposed individuals are likely to carry chronic asymptomatic $P$. falciparum infections (Sturrock et al., 2013; Ouédraogo et al., 2016), a situation in which the majority of this population can act as a reservoir for malaria transmission.

Our results showed 15 women with sub-microscopic infection in the cord samples with no evidence of infection in peripheral and/or placental samples. The unique presence of certain parasites in the cord can be explained by parasite clearance in the placenta and / or maternal-foetal transfusions before or during birth (Riley et al. 2001).

The MSP-2 marker is very polymorphic. We detected 24 distinct 3D7 alleles and 20 distinct FC27 alleles. Similar results were observed in Gabon (Kassberger et al., 2002, Mayengue et al., 2004) in Senegal (Schleiermacher et al., 2001). The 3D7/MSP2 allele was predominant such as reported in the previous report in the same site (Francine et al., 2016). Similar results are observed in Senegal (Jafari-Guemouri et al., 2005), Sudan (Fadlelseed et al., 2017). In Gabon, the predominant allele was FC27/MSP2 (Kassberger et al., 2002, Mayengue et al., 2004). Allelic frequencies are very different from one family to another and the distribution of these alleles reveals the genetic variability present in the parasite population and might reflect the geographical variation of parasite genotype and the level of transmission.

Multi-clonal infection was detected respectively in 40 (35.1\%), 24 (34.3\%) and 32 (91.4\%) of the peripheral, placental and cord samples. These results are the characteristic of the stable malaria transmission area (Mayengue et al., 2004).

The MOI could be used in the evaluation of malaria interventions as it is an indicator of 
malaria infection in humans and may, to some extent, reflect transmission and immunity malaria (Smith et al., 1999). In this cohort the MOI is lower than that reported (about 1.6 $\mathrm{MOI}$ ) in the previous study in the same site (Francine et al., 2016) and in other malaria endemic area (Beck et al., 2001; Walker-Abbey et al., 2005). Furthermore, The low MOI could be explained by the use of impregnated mosquito nets and the degree of immunity. As a result, the low parasite density of this study, which results in low $\mathrm{MOI}$, can be observed, but this did not affect the diversity of the parasites in circulation (IbaraOkabande et al., 2012).

The high prevalence of asymptomatic malaria in young pregnant women and those in their first and second pregnancies than those in their third or more pregnancies have reported (Smith et al., 1999). With successive pregnancies, women acquire a gravidity-dependent anti-malarial immunity, resulting in a decrease in both prevalence and severity of infection (Smith et al., 1999). Studies have demonstrated a correlation between the $\mathrm{MOI}$ and parasite density as well as age of the study participants (Mayengue et al., 2004; Omer et al., 2011). In our study, MOI was associated with neither maternal age nor gravidity, which is in line with other study (Kassberger et al., 2002, Walker-Abbey et al., 2005). Age and the acquisition of pregnancy-related immunity do not appear to result in elimination of parasites or reduction of the number of different infecting strains. The SP doses have not reduced the $\mathrm{MOI}$. This might result from the carriage of SP-resistant parasites in such women as suggested earlier (Moussiliou et al., 2013, Koukouikila-Koussounda et al., 2015; Ojurongbe et al., 2018).

The cross-sectional nature of the study did not allow participants to be followed until delivery. The lack of current entomological data during the collection of samples and the lack of data on the use of mosquito nets by pregnant women do not allow us to deepen the analyses on the low multiplicity of infections.

\section{Conclusions}

In summary, this study showed that there is a high prevalence of sub-microscopic infection and a moderate genetic diversity of Plasmodium falciparum. Age, gravidity and doses of preventive treatment based on sulfadoxine-pyrimethamine do not interfere with the 
multiplicity of infections. These results contribute to the understanding of the dynamic of parasites in blood sample types during pregnancy and parasite population circulating in Congo.

\section{Funding}

This study was financially supported by Total E\&P Congo and YM received support from Campus France. YM received a financial training support from (CANTAM) RegNet 2015 funded by EDCTP and European member states. YM was funded by a grant from Campus France for his stays in Aix-Marseille Université.

\section{Competing interests}

The authors declare that they have no competing interests.

\section{Acknowledgements}

We are highly indebted to all women who donated blood samples for this study. We are also sincerely grateful to the staff of Madibou Integrated health center for their kind assistance. We thank Mr Benjamin Galloy, Mr Dagène Ebouroumbi and Ms Sylvia Nkombo-Nkoula for lab assistance. FN and FKK are members CANTAM (EDCTPRegNet2015-1045 ) and PANDORA-ID-Net (Grant Agreement RIA2016E-1609 ) networks supported by EDCTP and European member states.

\section{Data availability}

Raw data of the work presented here are available upon request.

\section{References}

Beck S, Mockenhaupt FP, Bienzle U, et al (2001) Multiplicity of Plasmodium falciparum infection in pregnancy. The American Journal of Tropical Medicine and Hygiene 65:631-636. doi: 10.4269/ajtmh.2001.65.631 
Cohee LM, Kalilani-Phiri L, Mawindo P, et al (2016) Parasite dynamics in the peripheral blood and the placenta during pregnancy-associated malaria infection. Malar $\mathrm{J}$ 15:483. doi: 10.1186/s12936-016-1541-x

Cottrell G, Moussiliou A, Luty AJF, et al (2015) Sub-microscopic Plasmodium falciparum Infections Are Associated With Maternal Anemia, Premature Births, and Low Birth Weight. Clin Infect Dis 60:1481-1488. doi: 10.1093/cid/civ122

Dellicour S, Tatem AJ, Guerra CA, et al (2010) Quantifying the number of pregnancies at risk of malaria in 2007: a demographic study. PLoS Med 7:e1000221. doi: 10.1371/journal.pmed.1000221

Duffy PE, Fried M (1999) Malaria during pregnancy: parasites, antibodies and chondroitin sulphate A. Biochem Soc Trans 27:478-482

Fadlelseed OE, Osman ME, Shamseldin NM, et al (2017) Plasmodium falciparum genotypes in matched peripheral, placental and umbilical cord blood in an area characterised by unstable malaria transmission in eastern Sudan. Heliyon 3:e00326. doi: 10.1016/j.heliyon.2017.e00326

Fleischhacker M (2001) The 2nd International Symposium on Circulating Nucleic Acids in Plasma and Serum (CNAPS-2), Hong Kong, February 20-21, 2001. Eur J Med Res 6:364-368

Francine N, Damien B, Anna F, et al (2016) Characterization of asymptomatic Plasmodium falciparum infection and its risk factors in pregnant women from the Republic of Congo. Acta Tropica 153:111-115. doi: 10.1016/j.actatropica.2015.10.009

Fried M, Duffy PE (1996) Adherence of Plasmodium falciparum to Chondroitin Sulfate A in the Human Placenta. Science 272:1502-1504. doi: 10.1126/science.272.5267.1502

Genton B, Betuela I, Felger I, et al (2002) A recombinant blood-stage malaria vaccine reduces Plasmodium falciparum density and exerts selective pressure on parasite populations in a phase 1-2b trial in Papua New Guinea. J Infect Dis 185:820-827. doi: $10.1086 / 339342$ 
Guitard J, Andersen P, Ermont C, et al (2010) Plasmodium falciparum population dynamics in a cohort of pregnant women in Senegal. Malaria Journal 9:165. doi: 10.1186/1475-2875-9-165

Hviid L (2011) The case for PfEMP1-based vaccines to protect pregnant women against Plasmodium falciparum malaria. Expert Review of Vaccines 10:1405-1414. doi: 10.1586/erv. 11.113

Ibara-Okabande R, Koukouikila-Koussounda F, Ndounga M, et al (2012) Reduction of multiplicity of infections but no change in $\mathrm{msp} 2$ genetic diversity in Plasmodium falciparum isolates from Congolese children after introduction of artemisinincombination therapy. Malaria Journal 11:410. doi: 10.1186/1475-2875-11-410

Jafari-Guemouri S, Ndam NT, Bertin G, et al (2005) Demonstration of a High Level of Parasite Population Homology by Quantification of Plasmodium falciparum Alleles in Matched Peripheral, Placental, and Umbilical Cord Blood Samples. Journal of Clinical Microbiology 43:2980-2983. doi: 10.1128/JCM.43.6.2980-2983.2005

Kamwendo' D, Dzinjalamala' K, Kanjala' CC, et al Plasmodium falciparum: PCR detection and genotyping of isolates from peripheral, placental, and cord blood of pregnant Malawian women and their infants.

Kassberger F, Birkenmaier A, Khattab A, et al (2002) PCR typing of Plasmodium falciparum in matched peripheral, placental and umbilical cord blood. Parasitology Research 88:1073-1079. doi: 10.1007/s00436-002-0715-3

Koukouikila-Koussounda F, Bakoua D, Fesser A, et al (2015) High prevalence of sulphadoxine-pyrimethamine resistance-associated mutations in Plasmodium falciparum field isolates from pregnant women in Brazzaville, Republic of Congo. Infection, Genetics and Evolution 33:32-36. doi: 10.1016/j.meegid.2015.04.007

Maïga-Ascofaré O, Rakotozandrindrainy R, Girmann M, et al (2015) Molecular epidemiology and seroprevalence in asymptomatic Plasmodium falciparum infections of Malagasy pregnant women in the highlands. Malaria Journal 14:. doi: 10.1186/s12936-015-0704-5 
Mayengue PI, Rieth H, Khattab A, et al (2004) Sub-microscopic Plasmodium falciparum infections and multiplicity of infection in matched peripheral, placental and umbilical cord blood samples from Gabonese women. Tropical Medicine and International Health 9:949-958. doi: 10.1111/j.1365-3156.2004.01294.x

Mockenhaupt FP, Rong B, Till H, et al (2000) Sub-microscopic Plasmodium falciparum infections in pregnancy in Ghana. Tropical Medicine and International Health 5:167173. doi: 10.1046/j.1365-3156.2000.00532.x

Moussiliou A, De Tove YS-S, Doritchamou J, et al (2013) High rates of parasite recrudescence following intermittent preventive treatment with sulphadoxine-pyrimethamine during pregnancy in Benin. Malaria Journal 12:195. doi: 10.1186/1475-2875-12-195

Nosten F, Rogerson SJ, Beeson JG, et al (2004) Malaria in pregnancy and the endemicity spectrum: what can we learn? Trends in Parasitology 20:425-432. doi: 10.1016/j.pt.2004.06.007

Ntoumi F, Vouvoungui JC, Ibara R, et al (2013) Malaria burden and case management in the Republic of Congo: limited use and application of rapid diagnostic tests results. BMC Public Health 13:. doi: 10.1186/1471-2458-13-135

Ojurongbe O, Nguetse CN, Fayemiwo SA, et al (2018) High prevalence of dihydrofolate reductase gene mutations in Plasmodium falciparum parasites among pregnant women in Nigeria after reported use of sulfadoxine-pyrimethamine. Pathogens and Global Health 112:86-92. doi: 10.1080/20477724.2017.1422615

Okell LC, Ghani AC, Lyons E, Drakeley CJ (2009) Sub-microscopic Infection in Plasmodium falciparum -Endemic Populations: A Systematic Review and MetaAnalysis. The Journal of Infectious Diseases 200:1509-1517. doi: 10.1086/644781

Omalu ICJ, Mgbemena C, Mgbemena A, et al (2012) Prevalence of Congenital Malaria in Minna, North Central Nigeria. Journal of Tropical Medicine 2012:1-5. doi: 10.1155/2012/274142 
Omer S, Khalil E, Ali H, Sharief A (2011) Sub-microscopic and multiple plasmodium falciparum infections in pregnant Sudanese women. N Am J Med Sci 3:137-141. doi: 10.4297/najms.2011.3137

Ouédraogo A, Tiono AB, Diarra A, et al (2012) Transplacental Transmission of Plasmodium falciparum in a Highly Malaria Endemic Area of Burkina Faso. Journal of Tropical Medicine 2012:1-7. doi: 10.1155/2012/109705

Ouédraogo AL, Gonçalves BP, Gnémé A, et al (2016) Dynamics of the Human Infectious Reservoir for Malaria Determined by Mosquito Feeding Assays and Ultrasensitive Malaria Diagnosis in Burkina Faso. J Infect Dis 213:90-99. doi:

10.1093/infdis/jiv370

Riley EM, Wagner GE, Akanmori BD, Koram KA (2001) Do maternally acquired antibodies protect infants from malaria infection? Parasite Immunology 23:51-59. doi: 10.1046/j.1365-3024.2001.00364.

Saute F, Menendez C, Mayor A, et al (2002) Malaria in pregnancy in rural Mozambique: the role of parity, sub-microscopic and multiple Plasmodium falciparum infections. Tropical Medicine and International Health 7:19-28. doi: 10.1046/j.13653156.2002.00831.x

Smith T, Beck HP, Kitua A, et al (1999) Age dependence of the multiplicity of Plasmodium falciparum infections and of other malariological indices in an area of high endemicity. Trans R Soc Trop Med Hyg 93 Suppl 1:15-20

Steketee RW, Wirima JJ, Campbell CC (1996) Developing effective strategies for malaria prevention programs for pregnant African women. Am J Trop Med Hyg 55:95-100

Sturrock HJW, Hsiang MS, Cohen JM, et al (2013) Targeting Asymptomatic Malaria Infections: Active Surveillance in Control and Elimination. PLoS Medicine 10:e1001467. doi: 10.1371/journal.pmed.1001467

Snounou G, Viriyakosol S, Xin Ping Zhu, et al (1993) High sensitivity of detection of human malaria parasites by the use of nested polymerase chain reaction. Mol Biochem Parasitol 61: 315-20. doi: 10.1016/0166-6851(93)90077-B 
Takala SL, Coulibaly D, Thera MA, et al (2007) Dynamics of Polymorphism in a Malaria Vaccine Antigen at a Vaccine-Testing Site in Mali. PLoS Medicine 4:e93. doi: 10.1371/journal.pmed.0040093

Takala SL, Plowe CV (2009) Genetic diversity and malaria vaccine design, testing and efficacy: preventing and overcoming 'vaccine resistant malaria.' Parasite Immunology 31:560-573. doi: 10.1111/j.1365-3024.2009.01138.x

Trape JF and Zoulani A (1987). Malaria and urbanization in central Africa: the example of Brazzaville. Part II: Results of entomological surveys and epidemiological analysis. Trans. R. Soc. Trop. Med. Hyg. 81(suppl 2), 10-18.

Tshibola Mbuyi ML, Bouyou-Akotet MK, Mawili-Mboumba DP (2014) Molecular Detection of Plasmodium falciparum Infection in Matched Peripheral and Placental Blood Samples from Delivering Women in Libreville, Gabon. Malaria Research and Treatment 2014:1-6. doi: 10.1155/2014/486042

Walker-Abbey A, Djokam RR, Eno A, Leke RF, Titanji VP, Fogako J, Sama G, Thuita LH, Beardslee E, Snounou G, Zhou A, Taylor DW. (2005) Malaria in pregnant Cameroonian women: the effect of age and gravidity on submicroscopic and mixedspecies infections and multiple parasite genotypes. Am J Trop Med Hyg. 72(3):22935.

WHO, 2005. World Malaria report, 2005. World Health Organization https://apps.who.int/iris/bitstream/handle/10665/43213/9241593199_eng.pdf;jsessionid=07 2CD8222AB26F16289AB555A435027D?sequence $=1$ 